\author{
S. T. Schindera \\ M. Streit \\ U. Kaelin \\ E. Stauffer \\ L. Steinbach \\ S. E. Anderson
}

\section{Stewart-Treves syndrome: MR imaging of a postmastectomy upper-limb chronic Iymphedema with angiosarcoma}

Received: 13 March 2004

Revised: 22 April 2004

Accepted: 22 April 2004

Published online: 30 June 2004

(C) ISS 2004

S. T. Schindera ( $)$ - S. E. Anderson Department of Diagnostic Radiology, University Hospital of Bern,

3010 Inselspital, Bern, Switzerland e-mail: sebastian.schindera@insel.ch

Tel.: +41-31-6322435

Fax: +41-31-6324874

M. Streit · U. Kaelin

Department of Dermatology,

University Hospital of Bern,

Inselspital, Bern, Switzerland

\section{E. Stauffer}

Department of Pathology,

University Hospital of Bern,

Inselspital, Bern, Switzerland

L. Steinbach

Department of Radiology,

University of California San Francisco,

San Francisco, CA, USA

\begin{abstract}
The rare occurrence of angiosarcoma in postmastectomy upper-limb lymphedema with magnetic resonance (MR) imaging is discussed. Unfamiliarity with this aggressive vascular tumor and its harmless appearance often leads to delayed diagnosis. Angiosarcoma complicating chronic lymphedema may be low in signal intensity on T2weighting and short tau inversion recovery (STIR) imaging reflecting the densely cellular, fibrous stroma, and sparsely vascularized tumor histology. Additional administration of intravenous contrast medium revealed significant enhancement of the tumorous lesions. Awareness of angiosarcoma and its MR imaging appearance in patients with chronic lymphedema may be a key to early diagnosis or allow at least inclusion in the differential diagnosis.
\end{abstract}

\author{
Keywords Upper extremity · \\ Chronic lymphedema . \\ Angiosarcoma $\cdot$ Stewart-Treves \\ syndrome $\cdot$ MR imaging
}

\section{Introduction}

Lymphangiosarcoma of the upper limb was first described in postmastectomy patients by Stewart and Treves in 1948 [1]. It is today a rare but still potentially highly lethal vascular tumor occurring most often in chronic lymphedema due to radical mastectomy. Other risk factors of lymphedema that have been reported in association with angiosarcoma include trauma, filarial infection, and idiopathic acquired lymphedema [2].

Unfamiliarity with this malignancy and the often innocuous appearance may lead to delayed diagnosis. We describe a case of upper-extremity angiosarcoma developing after mastectomy with emphasis on magnetic res- onance (MR) imaging, which has rarely been reported in the world radiology literature $[3,4]$. The unusual features of our case are the subcutaneous location and multiplicity of the tumorous lesions as well as the decreased signal intensity on T2-weighted images of a sarcoma that histologically turned out to be an angiosarcoma.

\section{Case report}

An 80-year-old woman underwent a modified radical mastectomy and axillary node dissection on the right side for a T4aNOM0 invasive breast cancer. Postoperatively, the patient was irradiated with a total dose of 58 Gy. Chronic lymphedema of the right upper 


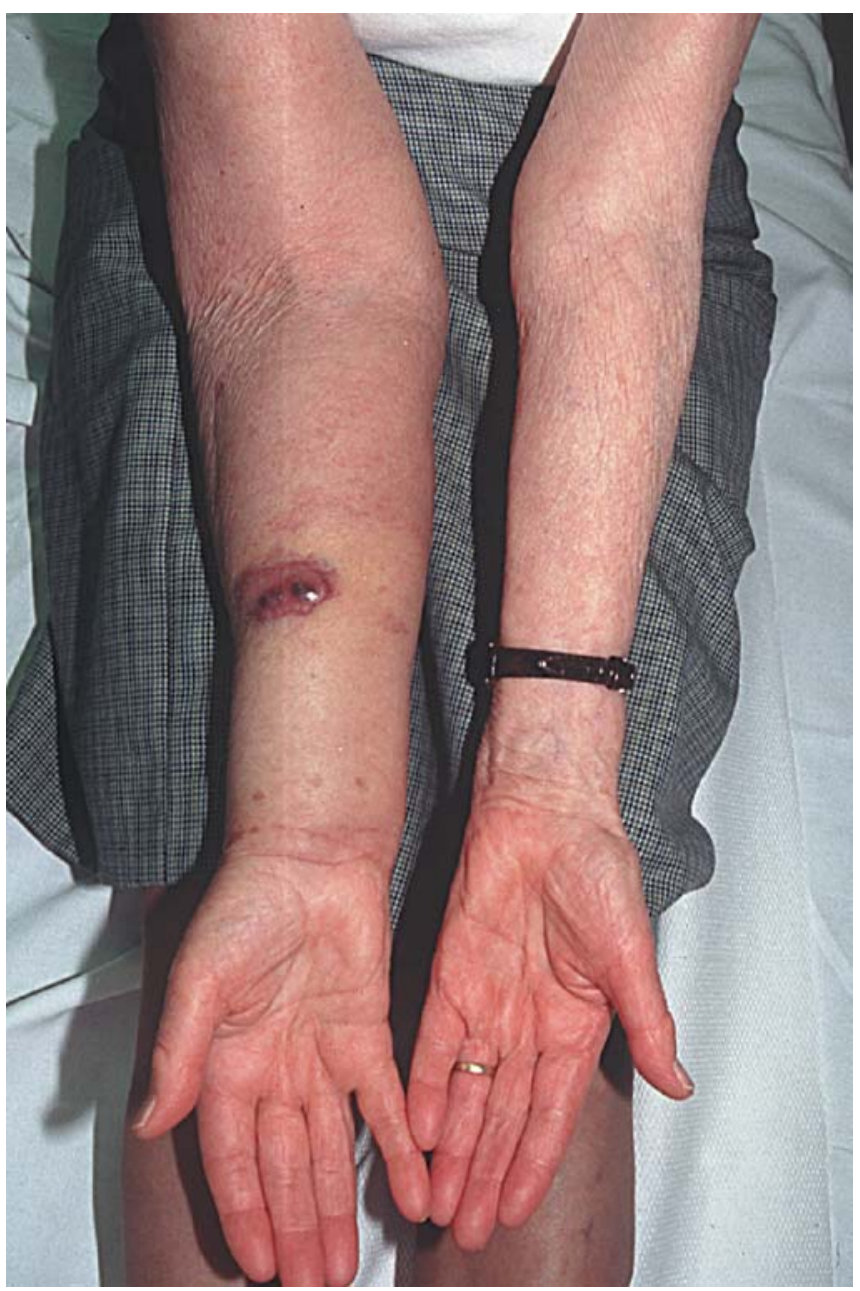

Fig. 1 Appearance of the larger lesion on the dorsum of the right forearm with chronic lymphedema compared to the normal appearance of the left arm

limb developed shortly after radiation therapy (Fig. 1). Nine years after mastectomy, the patient presented with a solid blue-reddish nodular lesion on the dorsum of her right forearm measuring approximately $3 \times 6 \mathrm{~cm}$. (Fig. 1). The tumorous lesion had grown rapidly within 2 months. Physical examination revealed a second satellite nodule of $0.8 \mathrm{~cm}$ in diameter on the volar aspect of the forearm. Telangiectasias could be found on the massively swollen right arm and on the lateral aspect of the breast and back. There was no lymphadenopathy.

Histology of a punch biopsy from the center of the larger forearm lesion demonstrated a vascular tumor consisting of a mixture of spindle cells and vascular lumina deformed by pleomorphic endothelial cells (Fig. 2A,B). Immunohistochemistry was positive for CD31 and factor VIII antigen, confirming the diagnosis of angiosarcoma.

MR imaging was performed for local extent and compartment anatomy for a presumed malignant soft tissue sarcoma. Prior to scanning, a marker was placed adjacent to the smaller second lesions on the volar aspect of the forearm, since this is a part of our protocol for tumor imaging, particularly for small lesions. The axial MR images showed marked circumferential thickening of the subcutaneous region consistent with the known lymphedema with
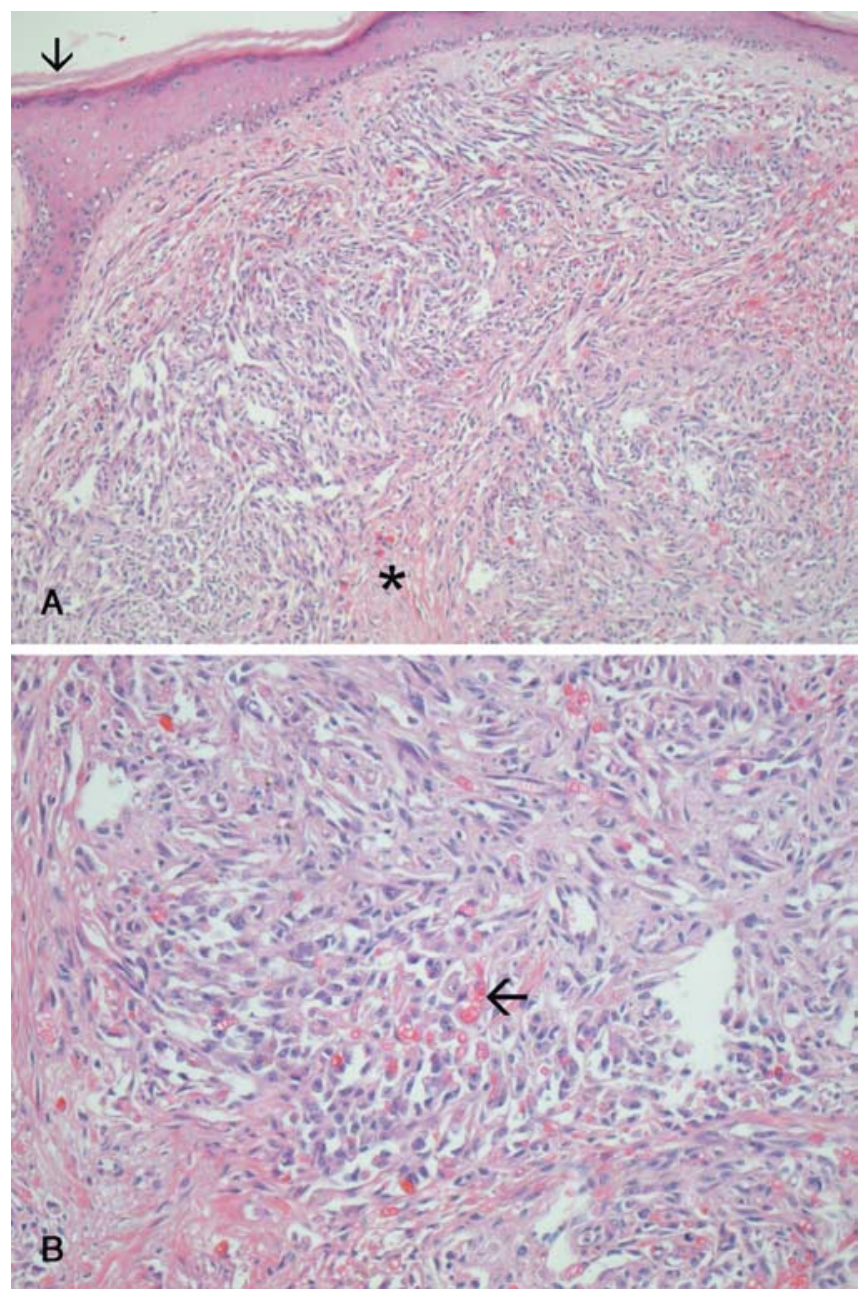

Fig. 2 A Hematoxylin-and-eosin-stained histopathology, $\mathrm{M} \times 10$, shows compact tumor nodule with very densely populated tumor cells. Prominent fibrous stroma is evident $(*)$. The skin surface is demarcated (arrow). B Hematoxylin-and-eosin-stained histopathology, $M \times 40$, shows evidence of small, fine, sparse vessels (e.g., arrow) with diffuse stroma surrounding

prominent skin thickening (Figs. 3A, 4A). There was no involvement of the deep fascia or muscles. The two dermal nodules were of decreased signal intensity on T1-weighted imaging compared with surrounding fat and isointense to muscle ( Figs. 3A, 4A). Corresponding $\mathrm{T} 2$-weighted fat-saturated images showed inhomogeneous predominantly decreased signal intensity compared to muscle (Figs. 3B, 4B). The larger nodule extended into the subcutaneous fat layer by $3 \mathrm{~mm}$, consistent with a more aggressive lesion (Fig. 3A). The second smaller lesion was more evident on T2weighting and after intravenous contrast enhancement and remained within the dermis. The obvious cutaneous discoloration and tumorous mass together with the findings of the MR images allowed easy identification of the larger tumor; however the smaller lesion was less easy to recognize. After intravenous administration of contrast medium (gadolinium dimeglumine), the two tumors enhanced significantly (Figs. 3D, 4C). In the remaining subcutaneous tissue, there was no evidence of mass formation or irregularity suggestive of additional tumors. 
Fig. 3 A Axial T1-weighted image (TR: 600, TE: 14) shows the large dermal lesion (white arrows) with extension into subcutaneous fat by $3 \mathrm{~mm}$ (black arrowheads). Also noted is the extensive circumferential skin and soft tissue thickening, consistent with the known lymphedema. B Corresponding axial FSE T2-weighted image (TR: 5300, TE: 98) shows that the tumor nodule (white arrows) is largely of low signal intensity. The tumor nodules margins were well-defined on T2 weighting (arrows). Adjacent thickened skin is noted (arrowheads). Again the extensive lymphedema is noted. The underlying muscle has a normal signal intensity. C Corresponding axial short tau inversion recovery (STIR) (T1:90, TR: 5680, TE: 15) shows the larger tumor nodule remains decreased in signal intensity. D Corresponding axial postgadolinium T1-weighted image (TR: 519, TE: 14) shows diffuse contrast enhancement within the tumor (arrows). Adjacent thickened skin contrast enhancement is noted (arrowheads)
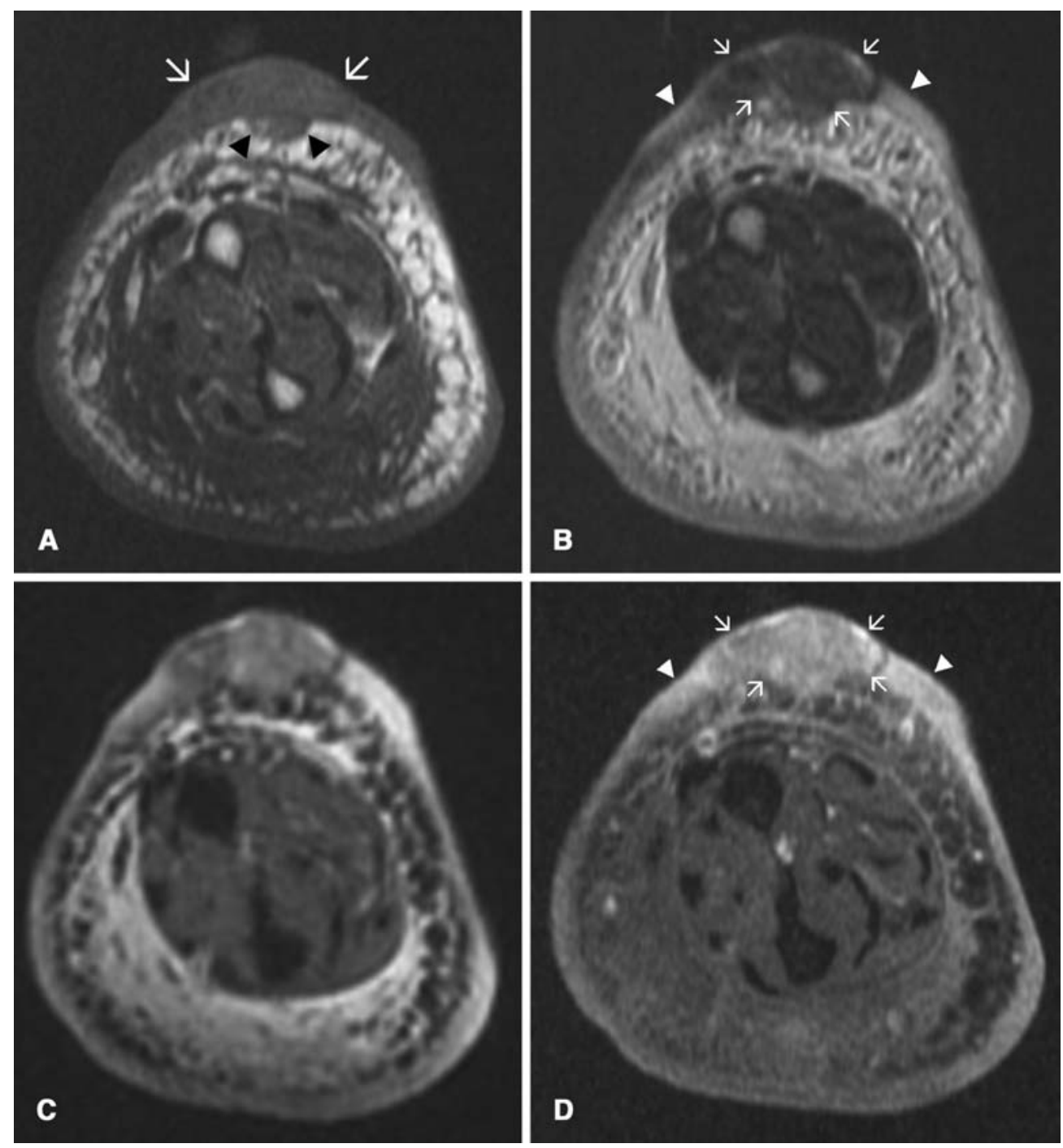

The patient insisted on limb salvage surgery and refused the proposed amputation. The tumorous mass on the forearm and the nodule on the volar aspect of the forearm were surgically removed with a margin of $1 \mathrm{~cm}$ and vertically deep to the level of the fascia. A mesh graft was transplanted with a very good functional result. Seven months after the excision of the tumor the patient was still free of recurrence or metastasis.

\section{Discussion}

Stewart-Treves syndrome (STS) was originally described as lymphangio-sarcoma of the upper extremity occurring many years after radical mastectomy for breast cancer [1]. The clinical appearance of the tumor according to the first descriptions of Stewart is "a slightly raised, macular or polypoid lesion . . . a solitary tumor followed by similar satellite areas that sometimes become confluent, forming a larger lesion" [5]. The tumors often appear as bluish-black, pink nodules on the edematous extremity, which tend to ulcerate and bleed. The differential diagnosis of lymphangiosarcoma includes Kaposi sarcoma, skin metastasis, malignant lymphoma, and deep infection of the skin.

Approximately $90 \%$ of the reported angiosarcomas involve the upper limb after radical mastectomy with or without radiotherapy [6]. Other rare causes for lymphangiosarcoma without prior breast carcinoma are trauma, infection, or idiopathic acquired lymphedema [2]. Lymphedema due to cardiac, hepatic, renal, or metabolic disease is not associated with a higher risk of developing a lymphangiosarcoma [6].

STS predominantly occurs in women. Only one case of a male patient with STS due to a postmastectomy lymphedema is reported [7]. The mean age of patients with STS ranges between the fifth and seventh decade [8]. The age correlates with the highest incidence of breast cancer.

In the 1960s, the incidence of STS varied from 0.07 to $0.45 \%$. Today, due to changes in surgical technique, STS 

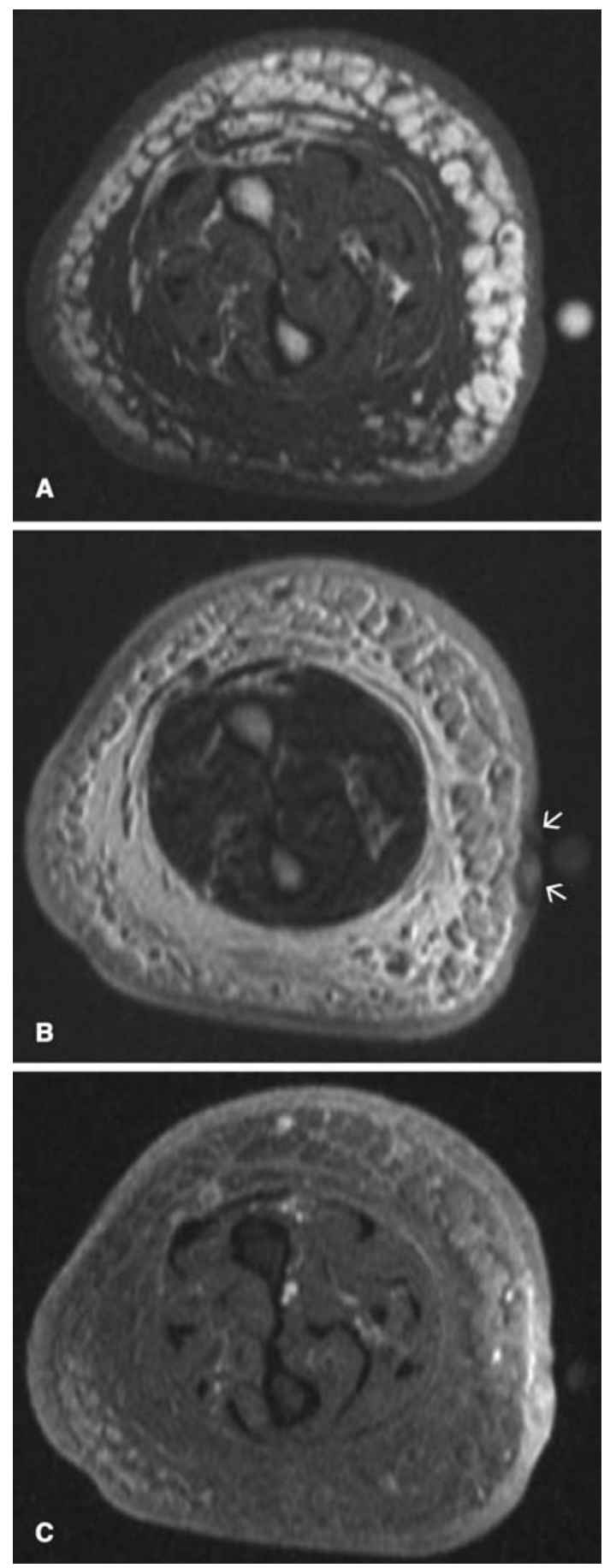

Fig. 4 A Axial T1-weighted image (TR: 600, TE: 14) with marker adjacent to the clinical reddish tumor nodule shows focal skin thickening and absence of subcutaneous involvement. B Corresponding axial FSE T2-weighted image (TR: 5300, TE: 90) shows the well-defined margins of the intradermal tumor (arrows), which measured $8 \mathrm{~mm}$ in length and was decreased in signal intensity. The extensive lymphedema of the subcutaneous region is noted as well as normal musculature. C Corresponding axial postgadolinium T1weighted image (TR: 519, TE: 14) shows significant enhancement of the nodule of the upper extremity is a very rare event. Less radical mastectomy, the avoidance of the combination of axillary dissection and radiation, as well as the increased application of sentinel node biopsy nowadays might lead to a further reduction of the incidence of postmastectomy angiosarcoma $[9,10]$.

The exact etiology of lymphangiosarcoma remains unclear. The tumor arises from vascular endotheliocytes and it is suggested that the malignancy is directly related to chronic lymphedema and an alteration in immune function [11]. The prevention of lymphedema might reduce the incidence of extremity angiosarcoma, and this is strongly recommended by many authors $[6,12]$. The mean latency period between the onset of the lymphedema and the appearance of the angiosarcoma is about 10-11 years [13].

Because of the rare occurrence of STS, there is little evidence on how lymphangiosarcoma should be optimally treated. Many authors believe that early surgical removal offers the best chance for prolonged survival; however, it is almost always followed by disease recurrence [2, 12, 14]. Other treatment strategies like external radiotherapy and chemotherapy (e.g., 5-fluorouracil, methotrexate, vincristine, bleomycin and vinblastine) are equally unsatisfactory. Both therapy options do not improve the overall long-term survival in patients with STS [12, 13, 14]. Isolated limb perfusion with $\mathrm{TNF} \alpha$ and melphalan proved to be efficient in a series of ten patients with multifocal lesions with $56 \%$ complete remission in a mean follow-up of 35 months [15]. However, it has to be emphasized that the survival of patients with STS is very poor, ranging from 19 to 31 months [14]. Most patients die of metastatic disease to the lungs [14]. Even if we lack evidence that the early recognition of lymphangiosarcoma is the key to a longer survival, a close follow-up of all patients with postmastectomy lymphedema is recommended.

MR is an established imaging modality in the investigation of accurate anatomical definition of peripheral lymphedema [16, 17]. Currently, there have been only two reports published on the MR appearance of angiosarcoma associated with chronic lymphedema of the lower extremity $[3,4]$. In our case, we present the MRbased morphological features of a patient with a postmastectomy upper limb angiosarcoma. The MR images revealed a diffuse dermal and subcutaneous circumferential edema involving the entire forearm, characteristic for a peripheral lymphedema. The two dermal nodules involving the subcutaneous region were isointense to muscle on T1-weighted and largely decreased signal intensity on T2-weighted images.

Nakazono et al. in 2000 described two cases of subcutaneous angiosarcomas of the lower limb associated with chronic lymphedema with MR imaging after hysterectomy [4]. Both tumors were isointense with muscle on $\mathrm{T} 1$ weighting and were largely decreased in signal intensity on T2 weighting. Interestingly, the nodules in 
our patient also had a decreased signal intensity on $\mathrm{T} 2$ and short tau inversion recovery (STIR) imaging (Fig. 3C). For a highly vascular malignant tumor, the decreased signal intensity on T2 weighting and STIR images is most unusual. Geographic areas of low signal intensity (short T2) have been described with fibrosarcoma, malignant fibrous histiocytoma, aggressive fibromatosis, and giant cell tumor of the tendon sheath [18]. The differential diagnosis of multiple soft tissue masses includes aggressive fibromatosis, desmoid tumors, neurofibromatosis, and Kaposi sarcoma [19]. However, these masses are easily distinguishable from our current case from the clinical and imaging appearance.

The second smaller lesion was evident with decreased signal on T2 weighting and was more prominently revealed after the administration of gadolinium. Therefore, the use of contrast medium in the setting of chronic lymphedema is recommended, even if it is of limited value for conspicuity and staging in musculoskeletal neoplasms [20].
After review of the MR images and the histological examination with the pathologist, we presume the low signal intensity was due to the relative sparsity of small vessels and the relative prominence of fibrous stroma with compact tumor cells. This agrees with the statement by Nakazono, who believes that the signal intensity on T2weighted images expresses the ratio between the vascular spaces and the tumor cells [4]. Gelmetti et al. reported a case of lymphangiosarcoma of the lower limb with high signal intensity on T2-weighted images [3]. However, there was no histological correlation.

In our case and in the two other cases from the literature, the postintravenous, contrast-enhanced, fat-saturated MR images demonstrated a marked contrast enhancement within the tumor nodule with well-delineated margins.

In conclusion, awareness of angiosarcoma and its appearance in patients with chronic lymphedema may allow for improved and accurate MR imaging preoperative diagnosis.

\section{References}

1. Stewart FW, Treves N. Lymphangiosarcoma in postmastectomy lymphedema: report of six cases on elephantiasis chirurgica. Cancer 1948; 1:64-81

2. Borel Rinkes IH, de Jongste AB. Lymphangiosarcoma in chronic lymphedema. Reports of 3 cases and review of the literature. Acta Chir Scand 1986; 152:227-230

3. Gelmetti W, Michalik-Himmelmann R, Leppek R, Klose KJ. Lymphedema-associated angiosarcoma (the StewartTreves syndrome) of the lower extremity: its MRT imaging. Rofo Fortschr Geb Rontgenstr Neuen Bildgeb Verfahr. 1996; 164:168-170

4. Nakazono T, Kudo S, Matsuo Y, Matsubayashi R, Ehara S, Narisawa H, Yonemitsu N. Angiosarcoma associated with chronic lymphedema (StewartTreves syndrome) of the leg: MR imaging. Skeletal Radiol 2000; 29:413416

5. Stewart NJ, Pritchard DJ, Nascimento AG, Kang YK. Lymphangiosarcoma following mastectomy. Clinical Orthopaedics and Related Research 1995; 320:135-141

6. Martin MB, Kon ND, Kawamoto EH, Myers RT, Sterchi JM. Postmastectomy angiosarcoma. Am Surg 1984; 50:541545

7. Oettle AG, van Blerk PJP. Postmastectomy lymphostatic endothelioma of Stewart and Treves in a male. Br J Surg 1963; 50:736-743
8. Lasa MV, Mateo P, Bascon N, Baquedano J, Fuertes F, Lopez P, Esco R. Lymphangiosarcoma in a chronic lymphedematous limb: a case report. Tumori 1995; 81:381-382

9. Kissin MW, Querci della Rovere G, Easton D, Westbury G. Risk of lymphoedema following the treatment of breast cancer. Br J Surg 1986; 73:580_ 584

10. Pezner RD, Patterson MP, Hill LR, Lipsett JA, Desai KR, Vora N, Wong JY, Luk KH. Arm lymphedema in patients treated conservatively for breast cancer: relationship to patient age and axillary node dissection technique. Int $\mathbf{J}$ Radiat Oncol Biol Phys 1986; 12:20792083

11. Futrell JW, Meyers GH. The regional lymphatics and cancer immunity. Ann Surg 1973; 177:1-11

12. Kaufmann T, Chu F, Kaufman R. Postmastectomy lymphangiosarcoma (Stewart-Treves Syndrome): report of two long-term survivals. Br J Radiol 1991; 64:857-860

13. Chen KT, Bauer V, Flam MS. Angiosarcoma in postsurgical lymphedema. An unusual occurrence in a man. Am J Dermatopathol 1991; 13:488-492
14. Malhaire JP, Labat JP, Simon H, Le Maux H, Spindler P, Lucas B, Lamezec B. One case of Stewart-Treves syndrome successfully treated at two years by chemotherapy and radiation therapy in a 73-year-old woman. Acta Oncol 1997; 36:442-443

15. Lans TE, de Wilt JH, van Geel AN, Eggermont AM. Isolated limb perfusion with tumor necrosis factor and melphalan for nonresectable StewartTreves lymphangiosarcoma. Ann Surg Oncol. 2002; 9:1004-1009

16. Case TC, Witte CL, Witte MH, Unger EC, Williams WH. Magnetic resonance imaging in human lymphedema: comparison with lymphangioscintigraphy. Magn Reson Imaging 1992; 10:549558

17. Haaverstad R, Nilsen G, Myhre HO, Saether OD, Rinck PA. The use of MRI in the investigation of leg oedema. Eur J Vasc Surg 1992; 6:124-129

18. Sundaram M, McGuire MH, Schajowicz F. Soft tissue masses: histologic basis for decreased signal (short T2) on T2-wheighted MR images. AJR 1987; 148:1247-1250

19. Kransdorf MJ, Murphey MD. Imaging of soft tissue tumors. Philadelphia: Saunders; 1997:48-49

20. Mirowitz SA, Totty WG, Lee JKT. Characterization of musculoskeletal masses using dynamic Gd-DTPA enhanced spin-echo MRI. J Comput Assist Tomogr 1992; 16:120-125 